\title{
Clinical and Genetic Characterization and Biochemical Correlation at Presentation in 48 Patients Diagnosed with Urea Cycle Disorders at the Hospital Juan P Garrahan, Argentina
}

Journal of Inborn Errors of Metabolism \& Screening 2021, Volume 9: e20200026 DOI: https://doi.org/10.1590/2326-4594JIEMS-2020-0026

\author{
Ana Clara Bernal1,* $@$, Maria Cruz Tubio',,", Carolina Crespo ${ }^{2}$ \\ and Hernán Diego Eiroa ${ }^{1}$
}

\begin{abstract}
The clinical and biochemical findings in a cohort of 51 patients with urea cycle disorders followed at the Hospital Garrahan, Buenos Aires, Argentina were analyzed at the time of diagnosis ( 3 female patients were excluded). Of this cohort, 13/48 patients had earlyonset (EO), 23/48 had late-onset (LO), and 12/48 had a different presentation because they had a family risk background (FRB) and had been diagnosed since they were born. The most frequent deficiency disorder was OTCD (65\%). The initial ammonium value was evaluated, being higher in the EO group, with a statistically significant difference when compared with LO and FRB. $15 / 48$ patients fell into a coma at the time of diagnosis, mean ammonia was $829.54 \mu \mathrm{mol} / \mathrm{L}$, and $33 / 48$ did not fell into a coma, the mean ammonium was of $159.3 \mu \mathrm{mol} / \mathrm{L}(\mathrm{p}=0.001)$. 15 patients died: $62 \% \mathrm{EO}, 22 \% \mathrm{LO}(\mathrm{p}=0.0216), 17 \%$ FRB. A molecular study was performed on 35 patients. Patients with EO presentation suffer the most severe forms and still have high morbimortality. On the other hand, LO forms are forms of less severity that are finally diagnosed as a result of one or more acute episodes.
\end{abstract}

\section{Keywords}

Urea cycle disorder, early onset, late onset, family background.

\section{Introduction}

The Urea Cycle Disorders (UCDs) are diseases comprehended in the Inborn Errors of Metabolism. The urea cycle is produced in the periportal hepatocytes and is responsible for the detoxification of ammonium and the main source of arginine, citrulline, and ornithine biosynthesis. It is made up of five enzymes and two transporters, therefore, the deficit of the enzymes that are part of the urea cycle (Figure 1) and their transporters are the so-called primary hyperammonemia [1] and define the Urea Cycle Disorders.

The UCD may be altered by any of the following deficiencies: deficiency of carbamoyl phosphate synthetase 1 (CPS1D, OMIM \# 237300); deficiency of ornithine transcarbamylase (OTCD, OMIM \# 311250); deficiency of argininosuccinic synthetase (ASSD) or citrullinemia type I (OMIM \# 215700); deficiency of argininosuccinic lyase (ASLD, OMIM \# 207900); and deficiency of arginase 1 (ARG1D, OMIM \# 207800). The deficiency of the mitochondrial ornithine/citrulline antiporter (ORNT1) produces the syndrome hyperornithinemia, hyperammonemia, homocitrullinemia (HHH, OMIM \# 603861) and the deficiency of the aspartate/glutamate transporter (CITRIN) produces what is known as deficiency of citrine or citrullinemia type II (OMIM \# 605814). Furthermore, the urea cycle requires the activity of the enzyme $\mathrm{N}$-acetylglutamate synthetase (NAGS, OMIM \# 237310) to produce the allosteric activator of CPS 1, N acetylglutamate (NAG), OMIM \#207800) [2-3], and the intramitochondrial flux of bicarbonate generated by carbonic anhydrase Va (CAVA, MIM* 114761) [4].

\footnotetext{
${ }^{1}$ Hospital Prof. Dr. Juan P. Garrahan, Servicio de Errores Congénitos del Metabolismo, Buenos Aires, Argentina.

${ }^{2}$ Hospital Prof. Dr. Juan P. Garrahan, Laboratorio de Biología Molecular, Servicio de Genética, Buenos Aires, Argentina.

*These authors contributed equally to this work.
}

Received November 29, 2020, and in revised form June 10, 2021. Accepted for publication June 14, 2021.

Corresponding Author:

Ana Clara Bernal, Email: anaclarabernal@gmail.com 


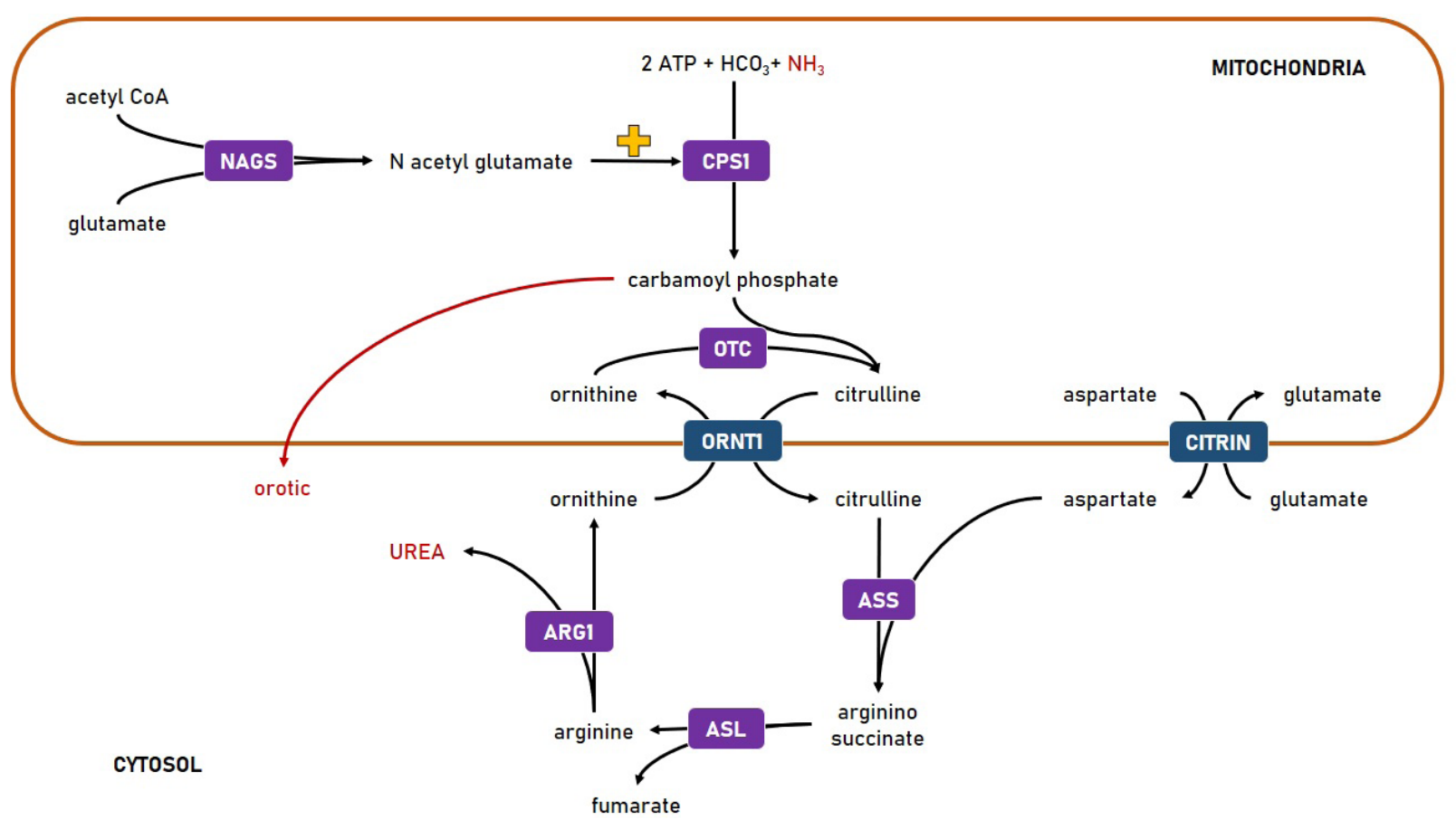

Figure 1. The Urea Cycle. ASL: Argininosuccinic lyase. ASS: Argininosuccinic synthetase. ARG1: Arginase 1. ATP: adenosine triphosphate. CITRIN: aspartate/glutamate transporter. CPS1: Carbamoyl phosphate synthetase 1. NAGS: N-acetylglutamate synthetase. ORNT1: Mitochondrial ornithine/ citrulline. OTC: Ornithine transcarbamylase.

Except for OTCD, which is linked to the X chromosome, all other enzyme deficiencies are autosomal recessive traits [5].

Currently, the incidence of UCD in Latin America is not known. There are few publications describing patients of Argentina, and they lack accurate information on the incidence of these diseases [6-9]. However, according to the European guidelines for the diagnosis and treatment of urea cycle disorders, the incidence is estimated to be 1: 8,000-1: 44,000 live births [10].

The UCDs present clear biochemical characteristics that allow their diagnosis. Hyperammonemia being the first. Hyperammonemia may be accompanied by liver failure, hepatitis, and respiratory alkalosis. Disease specific aminoacids profiles included elevation of citrulline, arginino succinic acid (ASA), arginine, ornithine (in combination with orotic aciduria), in distals UCDs (ASSD, ASLD, Arginase and HHH). Non-disease specific aminoacid abnormalities included elevated glutamine (all disorders) and reduce/absent citrulline (without orotic aciduria in NAGS and CPSD; with orotic aciduria in OTCD). The acute presentation shows altered levels of consciousness, acute encephalopathy, vomiting, and progressive poor appetite, multiorgan failure, liver failure, psychiatric symptoms. In the case of neonates, the characteristics are sepsis suspicion, hypertension [11], respiratory distress, hyperventilation, poor feeding, and vomiting. In reference to the chronic presentation, it is characterized by confusion, lethargy, ataxia, migrainelike headaches, protein aversions, vomiting, failure to thrive, liver failure, hepatomegaly, elevated liver enzymes, psychiatric symptoms, learning disabilities, and neurodevelopmental delay.
Rapid diagnosis and appropriate treatment are essential to reduce morbidity and mortality [12]. The treatment has two fundamental pillars, the restricted protein diet, supplemented with essential amino acids, vitamins, and trace elements [13] and the pharmacological treatment with ammonium scavengers (sodium benzoate, sodium phenylbutyrate) for the reduction of plasma ammonium concentrations, supplementation with intermediates of the urea cycle such as citrulline and arginine when there is a deficiency of them [14-17]. And, besides, in some cases, carglumic acid is required as an allosteric activator of the CPS 1 enzyme [18]. There are also dialysis treatments, which in current practice are started when conservative treatments fail or when ammonium values are excessively high. It has been observed that if dialysis is started with lower values of ammonia, the neurological impact will be less. In fact, in neonatal debut patients, all therapeutic options should be started without delay, including dialysis [19].

In Argentina, there is no neonatal screening for UCDs. Therefore, the diagnosis occurs as a result of clinical suspicion. The morbidity and mortality of the patient with this pathology depend on which was his or her age and stage of the disease at the time of diagnosis and thus to have the opportunity to start the appropriate medical treatment. And of course, of the form of the disease that the patient suffers.

Knowing the characteristics of the population under treatment, the starting point is to evaluate how the diagnostic and therapeutic strategies are carried out, and the sine qua non condition is to have the ability to implement changes that 
will subsequently achieve a positive impact on the outcome of patients. Consequently, our objective was to characterize clinically, temporarily, biochemically, and molecularly the population of patients with UCD diagnosed at the Hospital Juan P. Garrahan, Buenos Aires, Argentina, from January 2000 to December 2019, and to find out in our patient population what was the UCDs form of presentation.

\section{Material and Methods}

Retrospective information was collected from January 2000 to December 2019. The inclusion criteria were patients with a diagnosis of UCDs that had been clinically and biochemically characterized at the Juan P. Garrahan Hospital [20]. Both the clinical information and the biochemical results of the first reported episode of hyperammonemia were analyzed based on paper and computerized medical records. Asymptomatic women (relatives of known patients) with pathogenic variants in the OTC gene were not included in the study. All procedures performed in studies involving human participants should be in accordance with the ethical standards of the institutional and/or national research committee and with the Declaration of Helsinki of 1964, and its later amendments or comparable ethical standards.

\section{Age of Presentation and Diagnosis}

The patients were classified according to the enzyme deficiency diagnosed and according to their neonatal presentation. Earlyonset (EO) presentation included those patients with symptoms between 0 and 30 days of life, and late-onset (LO) presentation included those who had symptoms after 30 days of life. On the other hand, those patients in whom the presymptomatic diagnosis was made due to their family history were identified as family risk background (FRB) patients. In the case of earlyonset patients, the diagnosis time defined as the time between the onset of symptoms and the diagnosis of the disease was analyzed. Regarding late-onset patients, we analyzed whether there was a delay in diagnosis, which was defined as not having arrived at a diagnosis during the first symptoms of the disease registered in the patients' medical history. Confirmation of the diagnosis of the specific UCD alteration was genetic in most cases. In those cases, without a positive genetic diagnosis in the patient or in the family, the diagnosis was based on the biochemical profile (hyperammonemia with decreased plasma citrulline and the presence of substantial urinary orotate for OTCD; elevated citrulline with no arginosuccinate in ASSD; and the finding of arginosuccinate in ASLD) (Supplementary Table).

\section{Family Background}

The family history of the patients in each group was evaluated. The two first groups were differentiated according to whether they had a urea cycle disorder or not, and whether the factor of having had a family history had an impact on the clinical presentation of the disease, the time of diagnosis, and the survival of the patient under follow-up. The third group included those patients that had a family history of the disease and the knowledge of this fact induced medical suspicion Therefore, we concluded that thanks to the existence of the affected family member, asymptomatic newborns were treated early, during the disease-free period, and the patients that we classified as FRB were detected (Supplementary Table).

\section{Biochemical Parameters at the Time of Diagnosis}

Multiple biochemical parameters were evaluated at the time of diagnosis. These were, ammonia $(\mathrm{N}<62 \mu \mathrm{mol} / \mathrm{L})$, aspartate aminotransferase (ASAT), $\mathrm{N}<58 \mathrm{IU} / \mathrm{L}$, and alanine aminotransferase transaminase (ALAT) $(\mathrm{N}<56 \mathrm{IU} / \mathrm{L})$. We have defined hepatitis as having those ASAT and/or ALAT values that double the normal value [21]. The prothrombin time (PT) value was analyzed, the altered value was defined to a value of less than $70 \%$ and the international normalized ratio (INR) values were also analyzed. We defined acute liver failure as moderate coagulopathy (INR $>1.5$ ) with encephalopathy or the presence of severe coagulopathy (INR $>2.0$ ) even in the absence of encephalopathy [21]. Plasma amino acids were analyzed in 32 patients (HPLC-BIOCHROM 30), specifically evaluating glutamine (normal range: 330-850 $\mu \mathrm{mol} / \mathrm{L}$ ), alanine (normal range: $140-450 \mu \mathrm{mol} / \mathrm{L}$ ), arginine (normal range: $12-110 \mu \mathrm{mol} / \mathrm{L}$ ), citrulline (normal range: $8-47 \mu \mathrm{mol} / \mathrm{L}$ ) and arginino succinic acid (ASA) (normal range: 0). In 16 patients, amino acids were analyzed utilizing a dried blood drop using the mass/mass tandem: alanine (normal range: $70-345 \mathrm{MU}$ ), arginine (normal range: 2-50 MU) and citrulline (normal range: 2-30 UM). The quantitative excretion of orotic acid was also evaluated using the analysis of urinary organic acids using gas chromatography coupled to mass spectrometry. Some determinations that can be read in the supplementary table were not measured at the started out but were measured during the follow-up, however, they are included because they are crucial for the diagnosis.

\section{Molecular Analysis}

Molecular analysis was performed on 35 patients. On 19 through a NGS (next-generation sequencing) panel including the following genes: NAGS, CPS1, ASL, ASS1, ARG1, SLC25A13, SLC25A15 and $O T C$; on 16 patients through PCR (polymerase chain reaction) amplification of OTC gene, followed by Sanger sequencing, and on one patient through PCR amplification and Sanger sequencing of ASS1 gene. Variants were described in relation to the following reference sequences: NM_000048.4 (ASL), NM_000050.4 (ASS1), NM_001875.5 (CPS1) and NM_000531.6 (OTC), according to the HGVS-nomenclature recommendations. Exome Aggregation Consortium (ExAC), Human Gene Mutation Database (HGMD) and ClinVar databases were used to search for previous reports of the detected genomic variants. Pathogenicity of novel variants was assessed using the following in silico algorithms: SIFT, Provean (http://provean.jcvi.org/genome_ submit_2.php?species=human), Polyphen2 (http://genetics. 
bwh.harvard.edu/pph2/) and Mutation Taster (http://www. mutationtaster.org/). Novel variants were classified according to the American College of Medical Genetics and Genomics (ACMG) guidelines for interpretation of sequence variants.

\section{Statistical Analysis}

Statistical analysis was performed with SPSS v.20 software. Mean and standard deviation was calculated for normally distributed data and median, minimum, and maximum for skewed distributions. Differences between means of two groups were compared by the t-test and of three or more groups by ANOVA. Differences between medians of two groups were compared by Mann-Whitney U test, and of three or more groups by Kruskal-Wallis test. Association between two categorical variables was assessed by Fisher's exact test. A $p$ value $<0.05$ was considered statistically significant.

\section{Results}

During the period studied, we diagnosed 51 patients with urea cycle disorder (36 families, 30 females, and 21 males) at the Hospital Juan P Garrahan, Buenos Aires, Argentina, although 3 asymptomatic OTCD females were excluded, they did not undergo treatment but received a clinical follow-up. Therefore, throughout this study, we always mentioned 48 patients (Supplementary Table).

The most frequent clinical manifestations at the onset of the disease were hyperammonemia, followed by vomiting. From the neurological standpoint, seizures, irritability, and ataxia predominated. At least 6 patients started out with acute liver failure.

In $13 / 48$ patients, the disease presentation was early-onset (EO) 2/13 CPS1D, 4/13 OTCD, 1/13 ASSD, 6/13 ASLD. Nine of them had no family history and 4 had the antecedent of a deceased brother without a diagnosis. In 23/48 patients the clinical presentation was of late-onset (LO). In 20/23 patients the diagnosis was OTCD while the remaining 3 had a diagnosis of ASSD. Fifteen of them had an unknown clinical history, and regarding the 8 patients with family antecedents, in $5 / 8$ the antecedent corresponded to a known diagnosis, and $3 / 8$ had had deceased brothers or uncles without a diagnosis. It should be mentioned that these 8 patients had a diagnosis of OTCD. 12/48 patients were part of the FRB group: 7/12 OTCD, 4/12 ASSD, 1 ASLD. These patients were those who, due to the knowledge of their family history, were referred to our center to be studied at birth and to anticipate the natural evolution of the disease. We could divide this group into 3 different subgroups thanks to the information obtained regarding the type of disease that the affected family member had, either by clinical information and or by molecular diagnosis. Thus, as we differentiated a subgroup of FRB patients who were considered to have had a form of spontaneous disease, EO we called them neonatal FRB, $(\mathrm{N}$ FRB). In this group, we included 6/12 patients (2 OTCD,
3 ASSD, 1 ASLD). We called a second subgroup that would have had a late-onset form of the disease, FRB late-onset (L FRB), (3 OTCD, 1 ASSD). And finally, we had a third subgroup made up of female OTC patients, whom we called F FRB. We know that when it comes to OTC in females, apart from the fact that neonatal symptoms are not usually described, the disease can behave differently in each patient, and therefore it cannot be predicted.

In our cohort, $79 \%$ of the patients with a family history had at least one deceased relative. In 11 patients, the medical history confirmed that the deceased relative suffered from the same pathology that affects the patient (3 ASSD, 1 ASLD, and 7 OTCD), while in the remaining 8 patients, it was presumed.

During the first 10 years of the evaluated (period 1), 16 patients were diagnosed, 4 of the EO, (1 ASSD, 3 ASLD) and $11 \mathrm{LO}$, all of them with a diagnosis of OTCD. While, a male OTCD patient belonged to the FRB subgroup, brother of a known OTCD patient, was detected.

In the last 10 years (period 2), 32 patients were diagnosed. $9 \mathrm{EO}$, and $12 \mathrm{LO}$ patients. Regarding FRB, 11 patients are worth mentioning because in 10 of the 11 patients the enzymatic defect of the affected relative was known, while in the remaining patient there was a high suspicion that the defect had been due to OTC enzyme deficiency since the patient's two brothers had died in the neonatal period and had had biochemical findings compatible with this diagnosis.

The most frequent enzyme deficiency in our cohort was OTCD. It affected 31 patients (65\%), 55\% were females, and $45 \%$ were males. $4 / 31$ were EO patients (13\%), all of them were males. In 20 of these 31 OTCD patients the presentation of the disease was late-onset. LO (65\%), (5 males, 15 females). And 7 of these 31 (22\%) patients belong to the FRB group (5 males and 2 females).

In $75 \%$ of OTCD females, the onset of symptoms was before 2.9 years of age and they were diagnosed before 3.4 years. In males with OTCD, $75 \%$ presented symptoms before 0.9 years of age and were diagnosed before 2.2 years of age.

Only one of the females with late presentation form of the disease had no delay in diagnosis, while in the others the delay was between 27 and 1737 days. In male patients, with late presentation form, there was also a delay in reaching the diagnosis, from 60 to 1014 days.

ASS deficiency was diagnosed in $8 / 48$ patients (17\%). One patient had a neonatal EO presentation and 4 were classified as FRB. The onset of symptoms in $75 \%$ of the patients was before 1.3 years, and they were diagnosed before 1.6 years. Both in the patient with neonatal EO presentation of ASSD and in the 3 patients with delayed presentation of $\mathrm{LO}$, there was a delay in diagnosis of 38-91 days.

ASL deficiency was diagnosed in 7/48 patients (15\%), 6 of them with neonatal EO presentation and one with FRB. The diagnosis in this group of patients was made 2-6 days after the onset of symptoms.

CPS1 deficiency was diagnosed in two female patients (3\%). In in both of them the presentation was neonatal. The diagnosis 
was made in one case on the day of onset of symptoms and in the other 23 days after onset.

The age of onset of symptoms and the age of diagnosis were significantly lower in patients with ASSD and ASLD, compared with patients with OTCD (Supplementary Table). When dividing this last group according to gender, the median age of onset of symptoms and diagnosis in males was 2.5 and 9.5 days, respectively, while in females was 665 and 1064 days (21.8 and 34.8 months), respectively. Thus, there were no significant differences between the age of onset of symptoms and diagnosis between male patients with OTCD, ASSD, and ASLD, but there were between these and females with OTCD $(\mathrm{p}=0.005$ and $\mathrm{p}$ $=0.001$ respectively). No significant differences were found in the time of diagnosis between any of the groups.

Of the 13 EO patients, 4 of them had dead siblings. (2 OTCD, 2 ASLD). This fact anticipated the parents' consultation with the emergency system. $3 / 4$ patients were able to survive the onset of neonatal disease unlike their siblings, who despite not having a confirmed diagnosis, it was considered that they suffered the same condition. $95.7 \%$ of the patients with a late form of presentation were not diagnosed during the first episode. Even, of the 5 patients who had a history of known disease, only one was diagnosed during the first episode.

\section{Biochemical Parameters}

The biochemical parameters are described in (Table 1). The median ammonium values in the 48 patients were $218.9 \mu \mathrm{mol} / \mathrm{L}$ and that of glutamine, in 32 patients, $1207.5 \mu \mathrm{mol} / \mathrm{L}$. In patients with ASLD, the ammonium values were significantly higher compared to those in patients with OTCD and ASSD ( $\mathrm{p}=0.013$ and $\mathrm{p}=0.009$, respectively). Regarding glutamine levels, these were also higher in patients with ASLD, but the difference was significant only with those detected in patients with ASSD (Figure 2). The medians of ammonium and glutamine in male OTCD patients were $177 \mu \mathrm{mol} / \mathrm{L}$ and $1488 \mu \mathrm{mol} / \mathrm{L}$, and in female 188.8 $\mu \mathrm{mol} / \mathrm{L}$ and $1180 \mu \mathrm{mol} / \mathrm{L}$. For both parameters, the difference between the two groups was not significant.

The medians of ASAT and ALAT in the 40 patients were 60 IU/L and 43.5 IU/L. No significant differences were detected between the different enzyme deficiencies. Fourteen patients had hepatitis. Ten patients had a diagnosis of OTCD (2 EO/ $1 \mathrm{FRB} /$ 7 LO), three ASSD (1 EO/ 2 LO), one ASLD (1 EO).

The distribution of ammonium levels, stratified according to the period of presentation of the disease and its family history, are shown in (Figure 3).

It is observed that EO patients present higher ammonium values than patients in the remaining two groups $(\mathrm{p}<0.001)$. In turn, it is observed that the patients in the FRB group present similar ammonium values as the $\mathrm{LO}$. When dividing the group into N FRB and L RFB, it is observed that the ammonium values between the four groups are different $(\mathrm{p}<0.001)$, with the ammonium values in EO patients (mean: $1022.8 \mu \mathrm{mol} / \mathrm{L}$ ) significantly higher than in LO (mean: $194.4 \mu \mathrm{mol} / \mathrm{L}$ ) and L FRB (mean: $139.7 \mu \mathrm{mol} / \mathrm{L}$ ), but not than N FRB (mean: 505.1 $\mu \mathrm{mol} / \mathrm{L}$ ). Regarding glutamine, it could only be analyzed in two groups: patients with EO and LO diagnosis. Although there is a tendency in the EO group to have higher ammonium values, this difference is not statistically significant (Figure 3 ).

Table 1. UCDs patients and biochemical parameters.

\begin{tabular}{|c|c|c|c|c|c|c|}
\hline & & OTCD Male & OTCD Female & ASS1D & ASLD & CPS1D \\
\hline \multirow[t]{2}{*}{ Gender } & Female & - & 17 & 3 & 5 & 2 \\
\hline & Male & 14 & - & 5 & 2 & 0 \\
\hline \multirow[t]{2}{*}{ Age at diagnosis (days) } & Median & 9.5 & 1064 & 21 & 5 & - \\
\hline & Range & $0-1342$ & $2-3553$ & $0-678$ & $1-13$ & $1-23$ \\
\hline \multirow[t]{3}{*}{ Type of onset } & EO & 4 & - & 1 & 6 & 2 \\
\hline & LO & 5 & 15 & 3 & 0 & 0 \\
\hline & RFB & 5 & 2 & 4 & 1 & 0 \\
\hline \multirow[t]{3}{*}{ Ammonia $(\mu \mathrm{mol} / \mathrm{L})$} & $\mathrm{N}$ & 14 & 17 & 8 & 7 & 2 \\
\hline & Median & 177 & 188,8 & 144,55 & 1004,77 & - \\
\hline & Range & $74.34-1514.38$ & $67.26-466.69$ & $43.07-829.54$ & $149.86-1223.56$ & $354-1186.8$ \\
\hline \multirow[t]{3}{*}{ Glutamine $(\mu \mathrm{mol} / \mathrm{L})$} & $\mathrm{N}$ & 8 & 15 & 3 & 5 & 1 \\
\hline & Median & 1488 & 1180 & - & 2655 & - \\
\hline & Range & $730-4424$ & $230-3630$ & $241-900$ & $1086-2750$ & 720 \\
\hline \multirow[t]{3}{*}{ ASAT (UI/L) } & $\mathrm{N}$ & 11 & 16 & 7 & 5 & 1 \\
\hline & Median & 40 & 64 & 59 & 85 & 354 \\
\hline & Range & $24-410$ & $18-1860$ & $28-1157$ & $24-160$ & - \\
\hline \multirow[t]{3}{*}{ ALAT (UI/L) } & $\mathrm{N}$ & 11 & 16 & 7 & 5 & 1 \\
\hline & Median & 36 & 97.5 & 58 & 42 & 720 \\
\hline & Range & $9-183$ & $21-2051$ & $10-1320$ & $22-155$ & - \\
\hline
\end{tabular}



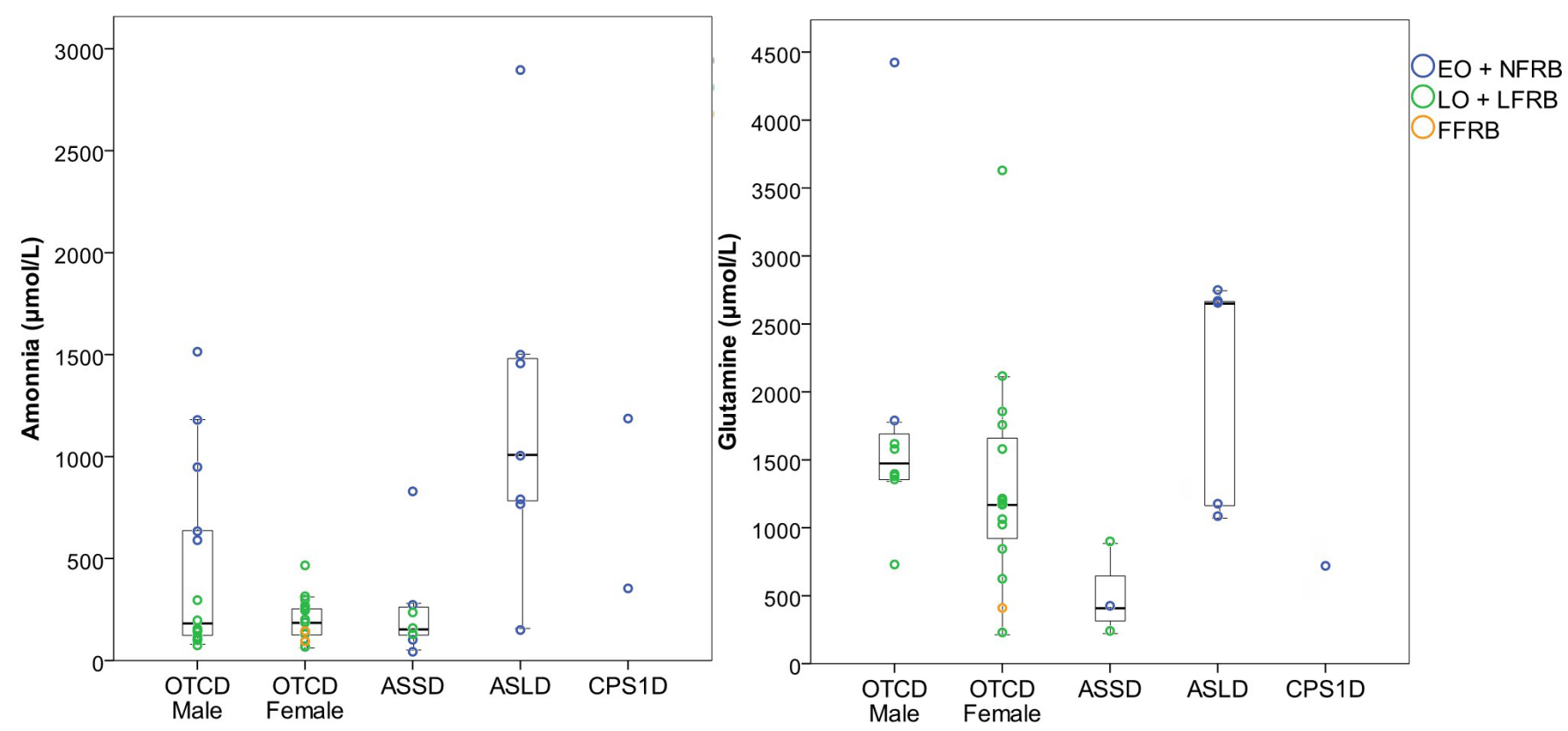

Figure 2. Ammonium and glutamine values according to enzyme deficiency. The distribution of the ammonium and glutamine values are shown in box plots for the OTC, ASS, and ASL deficiencies. Patients' values are indicated as dots, stratified by type of onset.

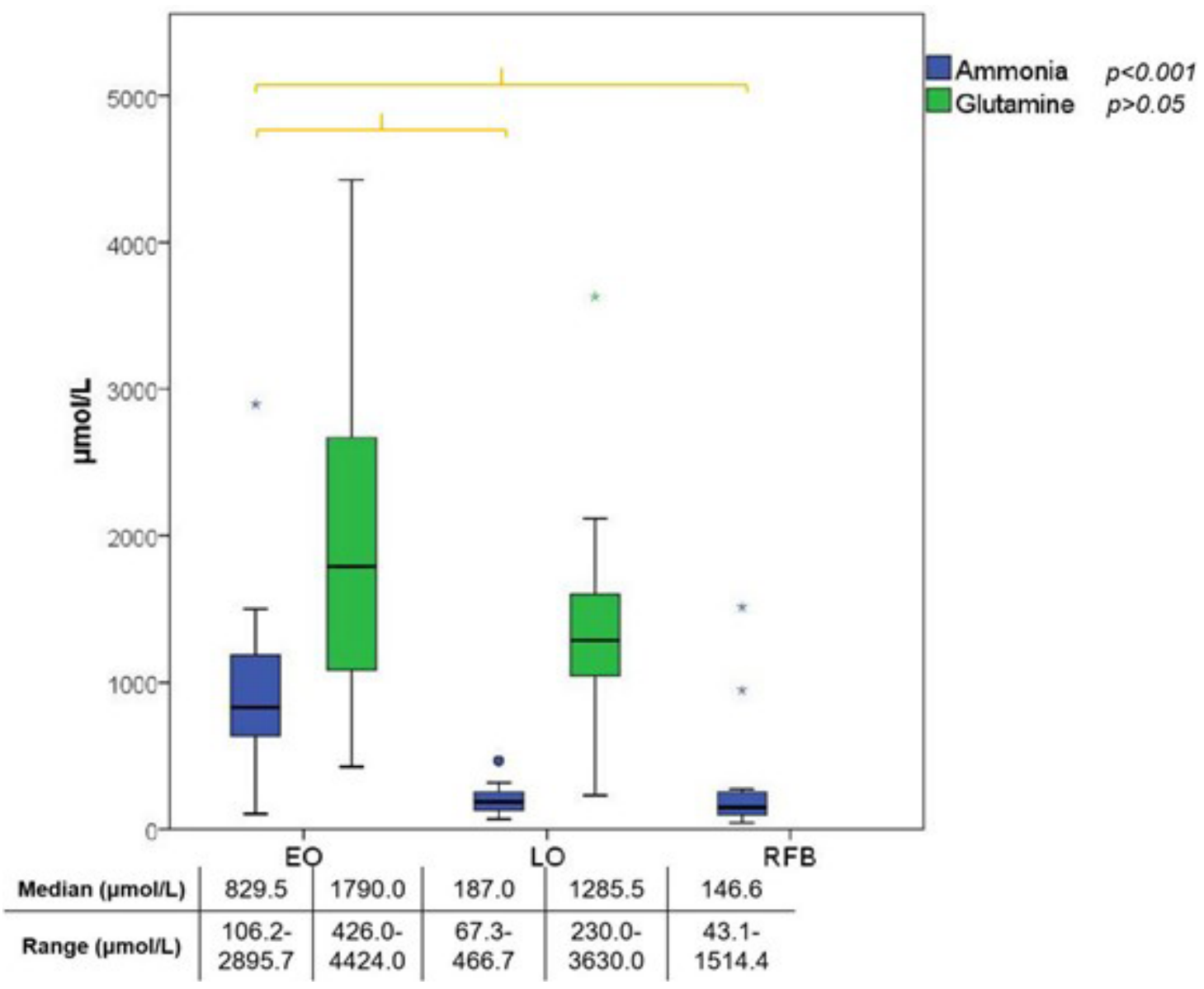

Figure 3. Ammonium and glutamine values according to the presentation of the disease. The distribution of the ammonium values are shown in box plots for the EO, LO and FRB patients, and the distribution of the glutamine values for the EO and LO patients. The braces indicate between which groups significant differences were detected in the post hoc tests. 


\section{Coagulogram}

An INR was measured in 13 patients (Supplementary Table). $2 / 13$ patients had INR between 1 and 1.5. In four patients INR was detected, they had 1.5 - 2, without suffering encephalopathy (Supplementary Table). And finally, in seven patients the INR was greater than 2, which is indicative of acute liver failure [21]. In this last group, 4 had a diagnosis of OTCD (3 late, $1 \mathrm{FRB}$ ), 2 of ASSD (1 LO, FRB), 1 of ASLD (EO).

\section{Coma}

In the present cohort, 15 patients fell into coma (31.2\%) (Supplementary Table). Of these, 8 had a diagnosis of OTCD (4 EO, 2 LO, 2 FRB), 2 ASSD (1 EO, 1 LO), 4 ASLD (all of EO diagnosis), and $1 \mathrm{CPSD}$ also EO. Median ammonium in patients who fell into coma as the first symptom was $829.54 \mu \mathrm{mol} / \mathrm{L}$ (range: $71.0-2895.72 \mu \mathrm{mol} / \mathrm{L})$, and in those who were not in a coma was $159.3 \mu \mathrm{mol} / \mathrm{L}$ (range: $43.07-1004.77 \mu \mathrm{mol} / \mathrm{L})(\mathrm{p}=0.001)$. Of the 13 patients with EO, 10 fell into coma (77\%) while of the $23 \mathrm{LO}$ patients, only 3 did so $(13 \%)(p=0.0006)$.

\section{Mortality}

Fifteen of the 48 patients died: 8/15 EO, 5/15 LO, 2/15 FRB (Supplementary Table).

We detected $8 / 15$ patients with EO. Four had OTCD, all of them were males, 2 of them were brothers and died during the neonatal period, their diagnosis was made retrospectively due to the birth of a third child, (the debut of the disease was in a hospital other than the J P Garrahan) 2 CPSD, 1 ASSD, 1 ASLD.

Only $2 / 15$ patients had FRB. Both patients had OTCD, one of them died during the neonatal period when he was six days old. The other patient had a severe neonatal presentation, despite treatment from the first day of life, he died at 6 months of life.

And finally, 5 were LO patients, 4 females, and 1 male, all with OTCD.

Of the 12 patients who died after 30 days of age, none did so at debut. 4 were males with OTCD, 3 were EO, and 1 was LO. 4 were females with OTCD, all with late presentation, then 1 ASSD, 1 ASLD, and the two other patients with CPS1D, all EO.

Of the 13 EO patients, 8 died (62\%), while of the 23 patients with LO, 5 died $(22 \%)(\mathrm{p}=0.0216)$. Patients with EO are 5.8 times more likely to die (95\% CI: 1.3 to 25.6$)$. FRB 2 died (17\%) both are severe forms of OTCD.

The survival rate of patients who survived after 30 days of life was $73 \%$, with a median follow-up of 9.3 years.

\section{Molecular Diagnostics}

The molecular study was carried out in 35 patients, it was confirmatory in 33 patients (94\%), and negative in 2: one patient with a biochemical diagnosis of OTCD and one with a diagnosis of ASLD. Twenty six different variants were detected in 4 genes, 14 in the OTC gene and 4 in each of the ASS1, ASL and CPS1 genes (Table 2). Ten of these variants were identified for the first time in this study. Of the total of variants detected, 19 were missense, 2 small deletions, one with an alteration in the reading frame and the other one conserved it. Two variants in splicing sites, an alteration of the first codon, and a variant of copy number. Three variants were detected in more than one family: the c.1168G> A variant in the ASS1 gene was identified in two patients in a homozygous state and in two in a heterozygous state, together with another variant, the c.386G $>\mathrm{A}$ and c.502C $>\mathrm{T}$ variants in the OTC gene were each detected in two unrelated patients, the latter being a novel variant.

\section{Discussion}

This report is the first large overview of data collected from UCDs patients from the Pediatric Hospital J P Garrahan. In this research, we analyzed the onset of the patients with UCDs diagnosed at the Hospital J. P. Garrahan during a period of 20 years. It is worth mentioning that a difference was found concerning the diagnoses made during the first half and the second half of that period. In the second period, $70 \%$ of the EO patients and $92 \%$ of the FRB patients of the cohort studied were diagnosed, maintaining a similar percentage of $\mathrm{LO}$ diagnosis in both periods. It was considered that this increase in the diagnosis of both the EO group and the FRB group is due to several factors: greater knowledge about the initial suspicion of metabolic diseases by pediatricians and neonatologists; training courses offered by the IEM service aimed at pediatricians and neonatologists in order to optimize consultation; the appearance of telemedicine; a more efficient and immediate communication system that allows consultation with the specialist to be framed and organized, becoming faster and more effective; and finally, the best follow-up and genetic counseling by the specialist to families who have had or have a member diagnosed with UCDs allow the study of the new family member during the disease-free period.

The number of LO patients remained constant in both periods, without the increase observed in the other groups, mainly due to the greater complexity of the diagnosis of this presentation, whose symptoms overlap with frequent clinical manifestations in pediatrics.

When we analyzed and distributed our cohort of patients in three groups: $\mathrm{EO}, \mathrm{LO}, \mathrm{FRB}$, we saw that all EO patients were diagnosed during the first episode. This was due to the severity of the neonatal presentation, which entails the performance of a greater number of studies, timely consultations, and even referral to more complex centers. The time from the onset of symptoms to diagnosis varied in the group of EO patients with a neonatal presentation from 0 days to 38 days. Those patients in whom the time to arrive at diagnosis was longer were those who had to be referred to a center of greater complexity for their diagnosis and treatment. Statistically significant differences were observed when comparing the time of diagnosis with the type of presentation since the time of diagnosis was longer in LO patients.

On the contrary, concerning LO patients, only one female patient was diagnosed at the time of the onset of the disease. It is 


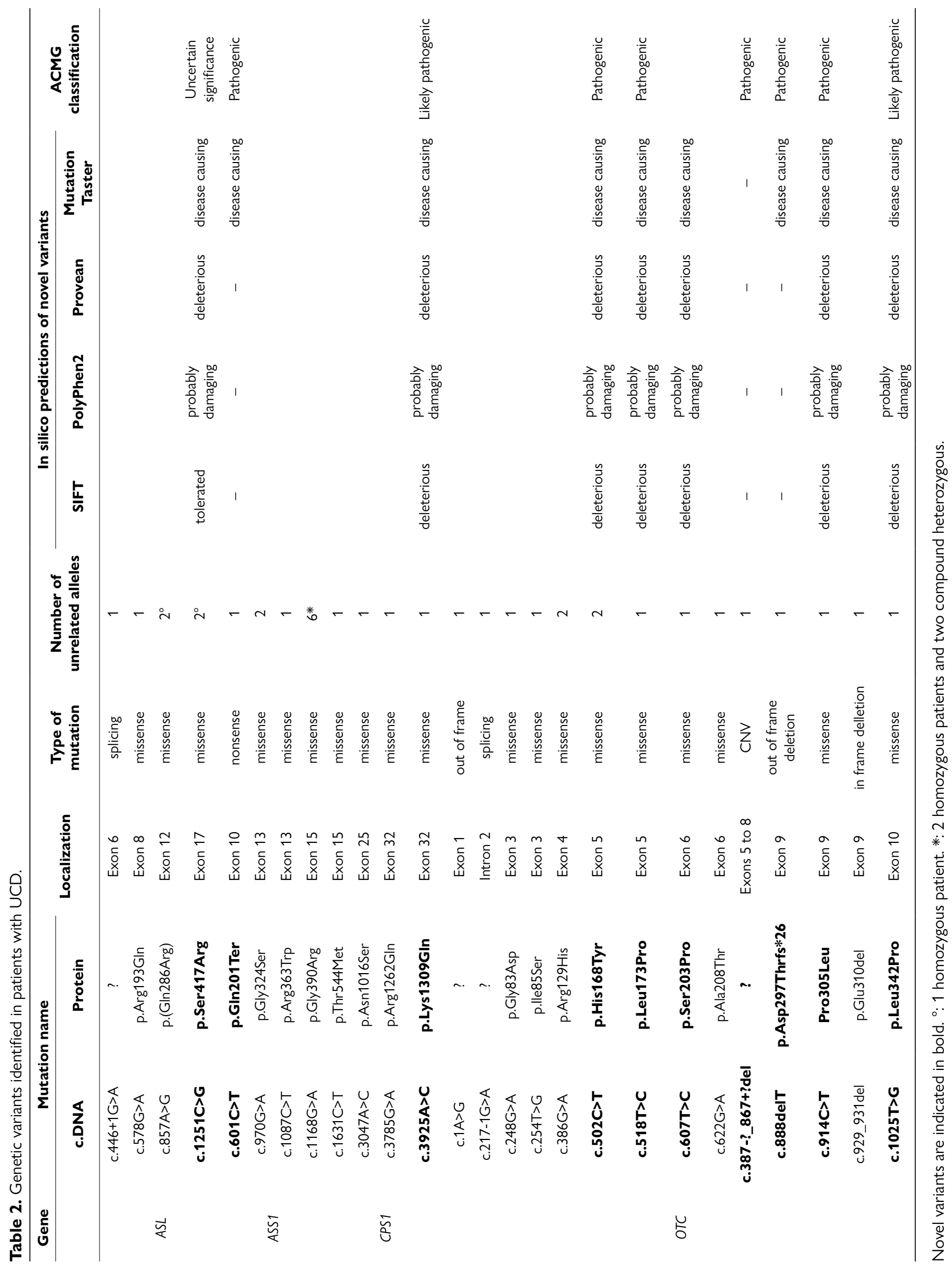


worth mentioning that it was her first episode of hyperammonemia, which was severe and required hospitalization. The patient had a known family history, cousins with OTCD, described in the current cohort.

Therefore, except for the recently described patient, in 22/23 LO patients there was a delay in the diagnosis of the disease. This meant that the patients were not diagnosed when they made their first medical consultation due to symptoms compatible with the UCDs, instead, they were diagnosed after this situation happened again and in the event of more severe episodes that had to be resolved through the intervention of the medical emergency, as had happened to the only patient previously described.

We consider that the delay in LO patients is due to different factors. In other words, symptoms can be more variable, symptoms can be attributable to other frequent pathologies in pediatrics, in addition to the fact that chronic UCDs are not conditions easily suspected by the pediatrician compared to other differential diagnoses, such as failure to thrive, psychiatric illness, cyclical vomiting syndrome, hepatitis or recurrent liver failure Usually, patients are not asked about dietary habits through a targeted questionnaire about protein aversions or fasting intolerance Hyperammonemic crisis are of lesser magnitude with mild or moderate clinical signs, added to the limited access of most hospitals in Argentina to basic biochemical studies for this type of pathology, such as, for example, the determination of plasma ammonia. This facilitates the lack of detection of the biochemical cornerstone of the UCDs. Ammonium testing is still considered a complex determination and not a frequently investigated determination.

All LO patients have been diagnosed in the context of hospitalization, which means that the symptoms resulting from the severity of the decompensation revealed the disease. Therefore, this shows that within the outpatient setting the suspicion of UCD as a differential diagnosis is extremely low. This statement is supported by the fact that all LO diagnoses were made during hospitalization.

When we considered the time of diagnosis and the type of presentation according to the enzymatic defect, it was evident that there were statistically significant differences only when comparing OTCD females with OTCD males, ASSD and ASLD. This difference is due to the inheritance pattern of OTCD, linked to X. Since in males, OTCD behaves with the same severity as in neonatal forms of the other enzyme deficiencies. While in females the symptoms may occur at any time from infancy to adulthood or may not appear in their entire life. This may be related to the degree of inactivation of X in heterozygous females [22]. Even in the same family, females can show wide clinical variability, from being asymptomatic to being refractory to medical treatment requiring liver transplantation [23].

On the other hand, we know that the moderate late-onset forms of ASSD and ASLD undergo fewer metabolic decompensations, with lower ammonium values, since they are distal enzymes. Therefore, making a diagnosis is more complex. The information obtained coincides with that observed in the bibliography [24].
Another way of arriving at a diagnosis of the late-onset forms is through the new-born screening test in those countries where it is performed. In Argentina, neonatal screening for defective enzymes such as ASS or ASL is not available. This could explain why, despite being ASLD, second in frequency worldwide [25], in our cohort it is the third in frequency and we only observed neonatal and severe forms.

When we analyzed the group of FRB patients, differentiated into N FRB, L FRB, and F FRB, we observed that all the patients, except one (number 34) (Supplementary Table), presented hyperammonemia despite the anticipated diagnosis, the timely referral to a tertiary care center, the early beginning of treatment, and the fact that the only situation of catabolic stress to which they were exposed was birth. All the patients began medical treatment with ammonia scavengers and a protein-restricted diet. This is why we consider that, except for F FRB patients who must be considered in each particular case, the remaining two forms of the disease should be treated from the moment of birth.

Six patients form part of the N FRB group and all have a history of deceased relatives with the same diagnosis. Two OTCD presented markedly high ammonium values (1514.38 and $948.6 \mu \mathrm{mol} / \mathrm{L}$ ), the highest in the group, despite the medical intervention performed. Evidently, these are patients with the classic neonatal presentation, which has a very high mortality even if they receive adequate medical treatment. One of these patients died within the neonatal period and the other after this period, at six months of life and with severe neurological sequelae as a result of the severity of the disease. Three ASSD, in whom early intervention before the onset of symptoms was essential for their survival since all their relatives had died in the neonatal period. One ASLD, due to timely treatment, presented an ammonium value of $149.86 \mu \mathrm{mol} / \mathrm{L}$, while her sister (patient $43 \mathrm{~h}$ ) presented a markedly elevated initial ammonium value of $1004.77 \mu \mathrm{mol} / \mathrm{L}$, died after the neonatal period, and had suffered severe neurological sequelae.

Four patients form part of group L FRB: 3 OTCD and one ASSD patient. All 3 OTCD patients present mutations compatible with LO OTCD and relatives with LO forms. The remaining patient in the group had a diagnosis of ASSD and a relative with a molecular diagnosis, included in the current cohort (patient number 35g). Although we knew that these were patients who had a form of LO disease, all the patients in this subgroup presented abnormal ammonium values, requiring medical treatment (pharmacological and dietary) from the neonatal period. Therefore, we consider that treatment during the neonatal period is beneficial, even in the case of $\mathrm{LO}$ disease.

Finally, the F FRB group consisted of 2 female OTCD patients. Both presented ammonium values similar to the patients classified within the group L FRB. This fact is consistent with the bibliography since it is not described that OTCD females develop severe neonatal forms. Both presented mutations not previously described. It was considered useful to study the OTCD females from the moment of birth since abnormal ammonium values were detected despite controlling the initial clinical exposure factors and with the administration of controlled 
dietary measures from birth. Both were defined as patients with the disease.

Regarding the specific analysis of the first identified ammonium value, interesting results were found concerning the three groups of patients. EO patients presented the highest ammonia values, as described in the bibliography. We observed that the LO and FRB groups of patients had lower ammonium values than the EO. Considering that we were evaluating $\mathrm{LO}$ forms of the disease, we included moderate or mild forms of this condition, which is reflected biochemically with lower ammonium values; and in the FRB group, the ammonium values found were due to the early beginning of the treatment, as previously described.

When we considered the more significant amino acid profiles for the diagnosis of the different UCDs, higher glutamine levels stand out in the group of ASLD patients, although the highest value was an outlier within the OTCD group. When comparing the glutamine values between the group of ASLD and ASSD patients, there were statistically significant differences that showed that the group of ASLD patients had the highest glutamine values. But when comparing the ASLD patient group with the OTCD group, no significant differences were found. This is explained, in the first place, because OTC deficiency is a proximal disorder, therefore, in comparison with subsequent enzymatic conditions, it presents higher ammonium values and, therefore, glutamine, both precursors of the urea cycle. Because the group of patients with OTCD was the largest and with the most variability in clinical and biochemical characteristics, not all the patients had such high levels of ammonium or glutamine. In contrast, all the patients diagnosed with ASLD had a neonatal presentation, with very high ammonium values, and these values were accompanied by very high glutamine values.

Liver function parameters were analyzed, specifically the INR, which allowed us to diagnose liver failure. Information on this biochemical parameter was not obtained in all patients, therefore, it was not feasible to show a trend. With the information obtained, a specific enzyme defect was not associated with liver failure. It is interesting to mention one patient (number 22f) with a diagnosis of late-onset OTCD who presented TP 16\% and coma. Although it is not the information presented in this article, it is worth mentioning that this patient belongs to one of the families of this cohort (family f), in which symptomatic females have recurrent liver failure regardless of adequate medical treatment and males die due to severe forms of neonatal onset. In this family, the genetic defect responsible for the pathology is the novel variant c.607T>C (p.Ser203Pro).

Regarding the data of the clinical manifestations at the beginning of the disease, we found information similar to the information that exists in the bibliography. It is important to highlight the value of coma, as a more serious manifestation and also because it is directly linked to the ammonium values that the patient presents. It was evident when analyzing the results that patients who fell into coma, as the initial symptom, presented higher ammonium values, therefore they were more at risk. Most were EO patients, $77 \%$ of the total. Once again, it shows that the forms of the neonatal disease are more severe. It should be mentioned that there were three patients that fell in coma in whom the ammonium values recorded did not agree with their clinical state. One possible explanation is that the biological samples were collected after medical treatment had been established. 2 N FRB patients presented coma, both OTCD, previously described, with extremely severe forms of the disease.

Concerning mortality, we found information that coincides with the bibliography. $62 \%$ of patients in the EO group died, reinforcing the assumption that this group presents the most severe forms of the disease. The mortality in the LO group was lower. It is important to highlight that in the FRB group the number of deaths was only 2 . These were the two OTCD patients with neonatal forms previously mentioned. We considered that this was due to early diagnosis and timely treatment before there was a suggestive clinical manifestation of the disease. It also showed better follow-up of families, better genetic counselling, and multidisciplinary work between the intensive care unit of the neonatology service and the specialist. Many of the patients, who had a deceased family relative with urea cycle disorder, had been referred to the Hospital J P Garrahan at the time of birth, and none of the late presentation patients died at the time of the onset of the disease.

In our cohort, in the evaluated time period the mortality rate was $31 \%$. On account of this, we assert that UDCs continue to be a group of diseases with high mortality despite currently available treatments, even with the possibility of performing liver transplantation as a healing treatment.

The molecular study allowed us to find the genetic cause of the enzymatic defect that generated the abnormal biochemical profile in $94 \%$ of the patients. The negative result in the patient with a diagnosis of OTCD may be due to the presence of deletions or duplications that represent a 5-10\% of the pathogenic variants described in OTC [26-27] and are not detected by the method used, especially in the case of female patients. The patient with a diagnosis of ASLD that was also negative could present deep intronic variants that affect RNA splicing and that are outside the regions covered by the method used, which includes exons and intronic flanking sequences. Among the previously reported variants detected in this cohort, c.1168G $>A$ (p.Gly390Arg) in ASS1 gene was the most frequent one. This is the most common variant found in patients with early onset phenotype of citrullinemia type I worldwide [28] and also in other subpopulations from our country [6-7]. The genotypephenotype correlation is described in patients with OTCD [26], and most of the variants can be classified as EO, LO or female, when they have not been detected in male patients. In the 9/12 patients with OTCD in whom the identified variant was reported, the presentation of the disease was correlated with that previously described. The remaining 3 patients had a family history and the presentation was neonatal, but the variants were previously related to late presentation. In the present study, 38\% of the variants identified in this study had not been previously reported in the bibliography. Three of them could be classified 
as pathogenic because of their nature: a nonsense variant in the ASS1 gene (patient 38 of Supplementary Table) and a gross deletion comprising exons 5 to 8 of the OTC gene (patient 16 of Supplementary Table) could also be labeled as highly severe variants because of the neonatal onset of both patients. The third one, an insertion, c.888delT (p.Asp297Thrfs26X), in the OTC gene, causing a frameshift, could be also classified as highly severe since it was detected in a female symptomatic OTCD patient. The rest of them were missense variants; according to the in silico analysis performed, all of them could be classified as probably damaging. In particular, c.607T $>C$ (p.Ser203Pro) was identified in several members of a family (f of Supplementary Table), with males that presented with EO and died and females with very severe manifestations, which would indicate the deleterious effect of this variant. Only one novel variant, c.502C > T (p.His168Tyr), was identified in two non related female OTCD patients with similar clinical outcomes between them.

\section{Conclusions}

We know that urea cycle disorders are diseases that present different severity levels.

Patients with EO presentation suffer the most severe forms, this fact might arise suspicion in neonatologists and thus avoid diagnosis delay. Although the existence of a diseasefree period is described, it is noteworthy that our patients in the FRB group, who did not present clinical symptoms, had abnormal ammonium values, possibly secondary to the catabolism typical of birth and required pharmacological and dietary treatment to normalize it. Early treatment of these patients during the symptom-free period decreases exposure to the primary intoxication factor. Adequate counseling and monitoring of families with UCD allow anticipating symptoms and effectively manage the patient's situation during the first hours of life, despite not having a prenatal diagnosis. In recent years, molecular studies and the detection of carriers contributed to genetic counseling. It is particularly useful in the case of OTCD since it allows detecting asymptomatic females in the proband's family at risk of having affected children and even anticipating symptoms that they may have. This practice has been improving over the last few years in the Inborn Errors of Metabolism division of the Hospital Garrahan.

On the other hand, in LO forms, it is observed that the delay in diagnosis prevails. Our LO patients had more than one consultation with the emergency services or with their family pediatrician due to compatible symptoms, but the diagnosis was inevitably reached during an acute decompensation that led to hospitalization with the consequent risk of life. This indicates that there is a lack of knowledge of physicians regarding the urea cycle disorders, therefore it is of the utmost importance to continue with the diffusion and training in this group of diseases, in which timely diagnosis can change the course of a patient's life.
Within this perspective, we consider that it would be important to add to the National Screening Program in Argentina the detection of ASSD, ASLD, and ARG1D.

\section{Acknowledgments}

The authors would like to thank the multidisciplinary team at the Inborn Errors of Metabolism in Hospital J P Garrahan, Buenos Aires, Argentina. We would also like to express our thanks to the following members of the Division of Genetics and Metabolism at the Children's National, Chief Dr. Marshall Summar, for their continuous support, encouragement, solidarity, and permanent collaboration; Dr. Kristina Patrice Cusmano-Ozog for her assistance with molecular data acquisition and validation; and Dr. Nicholas Ah Meh for his scientific advice and support. We also acknowledge Dr. Pablo Cafiero for his support and guidance. And finally, to Professor Rosa María Hermitte for the complete translation of the text, support, and commitment.

\section{Declaration of Conflicting Interests}

The author(s) declared no potential conflicts of interest with respect to the research, authorship, and/or publication of this article.

\section{Supplementary Material}

The following online material is available for this article: Supplementary Table - Clinical, molecular and biochemical characteristics of UCD patients.

\section{References}

1. Häberle J. Clinical and biochemical aspects of primary and secondary hyperammonemic disorders. Arch Biochem Biophys. 2013;536(2):101-108. doi:10.1016/j. abb.2013.04.009

2. Sancho-Vaello E, Marco-Marín C, Gougeard N, FernándezMurga L, Rüfenacht V, Mustedanagic M, Rubio V, Häberle J. Understanding N-Acetyl-L-Glutamate Synthase Deficiency: Mutational Spectrum, Impact of Clinical Mutations on Enzyme Functionality, and Structural Considerations. Hum Mutat. 2016;37(7):679-694. doi:10.1002/humu.22995

3. Mew NA, Caldovic L. N-acetylglutamate synthase deficiency: An insight into the genetics, epidemiology, pathophysiology, and treatment. Appl Clin Genet. 2011;4:127-135. doi:10.2147/TACG.S12702

4. Diez-Fernandez C, Rüfenacht V, Santra S, Lund AM, Santer $\mathrm{R}$, Lindner M, Tangeraas T, Unsinn C, et al. Defective hepatic bicarbonate production due to carbonic anhydrase VA deficiency leads to early-onset life-threatening metabolic crisis. Genet Med. 2016;18(10):991-1000. doi:10.1038/ $\operatorname{gim} .2015 .201$ 
5. Mew NA, Simpson KL, Gropman AL, Lanpher BC, Chapman KA, Summar ML. Urea cycle disorders overview definition of the urea cycle and clinical characteristics of urea cycle disorders definition. In: Adam MP, Ardinger HH, Pagon RA, et al., editors. GeneReviews. Seattle (WA): University of Washington, Seattle; 2019. p. 1-19.

6. Silvera-Ruiz SM, Arranz JA, Häberle J, Angaroni CJ, Bezard M, Guelbert N, Becerra A, Peralta F, et al. Urea cycle disorders in Argentine patients: clinical presentation, biochemical and genetic findings. Orphanet J Rare Dis. 2019;14(1):203. doi:10.1186/s13023-019-1177-3

7. Laróvere LE, Ruiz SMS, Angaroni CJ, de Kremer RD. Molecular epidemiology of citrullinemia type I in a risk region of Argentina: A first step to preconception heterozygote detection. JIMD Rep. 2012;6:27-29. doi:10.1007/8904_2012_127

8. Laróvere LE, Ruiz SMS, Arranz JA, de Kremer RD. Mutation spectrum and genotype-phenotype correlation in a cohort of Argentine patients with Ornithine Transcarbamylase Deficiency: a single-center experience. J Inborn Errors Metab Screen. 2018;6:1-5. doi:10.1177/2326409818813177

9. Bindi V, Eiroa H. Citrulinemia tipo I con insuficiencia hepática recurrente en un niño. Arch Argent Pediatr. 2017;115(1):e39-e42. doi:10.5546/aap.2017.e34

10. Häberle J, Burlina A, Chakrapani A, Dixon M, Karall D, Lindner M, Mandel H, Martinelli D, et al. Suggested guidelines for the diagnosis and management of urea cycle disorders: first revision. J Inherit Metab Dis. 2019;42(6):1192-1230. doi:10.1002/jimd.12100

11. Teufel U, Burgard P, Meyburg J, Lindner M, Poeschl J, Ruef P, Hoffmann G, Kölker S. High blood pressure, a red flag for the neonatal manifestation of urea cycle disorders. Orphanet J Rare Dis. 2019;14(1):80. doi:10.1186/s13023-019-1055-z

12. Gropman AL, Summar M, Leonard JV. Neurological implications of urea cycle disorders. J Inherit Metab Dis. 2007;30(6):865-879. doi:10.1007/s10545-007-0709-5

13. Holt LE Jr, Snyderman SE. Protein and amino acid requirements of infants and children. Nutr Abstr Rev. 1965;35:1-13.

14. Brusilow SW. Phenylacetylglutamine may replace urea as a vehicle for waste nitrogen excretion. Pediatr Res. 1991;29(2):147-150. doi:10.1203/00006450-19910200000009

15. Brusilow SW, Valle DL, Batshaw ML. New pathways of nitrogen excretion in inborn errors of urea synthesis. Lancet. 1979;2(8140):452-454. doi:10.1016/s01406736(79)91503-4

16. Rabier D, Kamoun P. Metabolism of citrulline in man. Amino Acids. 1995;9(4):299-316. doi:10.1007/BF00807268

17. Summar M. Current strategies for the management of neonatal urea cycle disorders. J Pediatr. 2001;138 (1 Suppl):S30-S39. doi:10.1067/mpd.2001.111834
18. Häberle J. Role of carglumic acid in the treatment of acute hyperammonemia due to $\mathrm{N}$-acetylglutamate synthase deficiency. Ther Clin Risk Manag. 2011;7:327-332. doi:10.2147/TCRM.S12703

19. Hediger N, Landolt MA, Diez-Fernandez C, Huemer $\mathrm{M}$, Häberle J. The impact of ammonia levels and dialysis on outcome in 202 patients with neonatal onset urea cycle disorders. J Inherit Metab Dis. 2018;41(4):689-698. doi:10.1007/s10545-018-0157-4

20. Hospital de Pediatría J P Garrahan. Available from: https:// www.garrahan.gov.ar/

21. Antiga LD (ed.). Pediatric hepatology and liver transplantation. Switzerland, Cham: Springer Nature; 2019. doi:10.1007/978-3-319-96400-3_28

22. Yorifuji T, Muroi J, Uematsu A, Tanaka K, Kiwaki K, Endo F, Matsuda I, Nagasaka K, et al. X-inactivation pattern in the liver of a manifesting female with ornithine transcarbamylase (OTC) deficiency. Clin Genet. 1998;54(4):349-453. doi: 10.1034/j.1399-0004.1998.5440415.x

23. Batshaw ML, Msall M, Beaudet AL, Trojak J. Risk of serious illness in heterozygotes for ornithine transcarbamylase deficency. J Pediatr. 1986;108(2):236-241. doi:10.1016/ s0022-3476(86)80989-1

24. Kölker S, Garcia-Cazorla A, Valayannopoulos V, Lund AM, Burlina AB, Sykut-Cegielska J, Wijburg FA, Teles EL, et al. The phenotypic spectrum of organic acidurias and urea cycle disorders. Part 1: the initial presentation. J Inherit Metab Dis. 2015;38(6):1041-1057. doi:10.1007/ s10545-015-9839-3

25. Tuchman M, Lee B, Lichter-Konecki U, Summar ML, Yudkoff M, Cederbaum SD, Kerr DS, Diaz GA, et al. Cross-sectional multi-center study of patients with urea cycle disorders in the United States. Mol Genet Metab. 2008;94(4):397-402. doi:10.1016/j.ymgme.2008.05.004

26. McCullough BA, Yudkoff M, Batshaw ML, Wilson JM, Raper SE, Tuchman M. Genotype spectrum of ornithine transcarbamylase deficiency: correlation with the clinical and biochemical phenotype. Am J Med Genet. 2000;93(4):313319. doi:10.1002/1096-8628(20000814)93:4<313::aidajmg11>3.0.co;2-m

27. Shchelochkov OA, Li F-Y, Geraghty MT, Gallagher RC, Van Hove JL, Lichter-Konecki U, Fernhoff PM, Copeland $S$, et al. High-frequency detection of deletions and variable rearrangements at the ornithine transcarbamylase (OTC) locus by oligonucleotide array CGH. Mol Genet Metab. 2009;96(3):97-105. doi:10.1016/j.ymgme.2008.11.167

28. Engel K, Höhne W, Häberle J. Mutations and polymorphisms in the human argininosuccinate synthetase (ASS1) gene. Human Mutat. 2009;30(3):300-307. doi:10.1002/ humu.20847 